\title{
FINITE ELEMENT INTERPOLATION OF NONSMOOTH FUNCTIONS SATISFYING BOUNDARY CONDITIONS
}

\author{
L. RIDGWAY SCOTT AND SHANGYOU ZHANG
}

\begin{abstract}
In this paper, we propose a modified Lagrange type interpolation operator to approximate functions in Sobolev spaces by continuous piecewise polynomials. In order to define interpolators for "rough" functions and to preserve piecewise polynomial boundary conditions, the approximated functions are averaged appropriately either on $d$ - or $(d-1)$-simplices to generate nodal values for the interpolation operator. This combination of averaging and interpolation is shown to be a projection, and optimal error estimates are proved for the projection error.
\end{abstract}

\section{INTRODUCTION}

The approximation of functions in Sobolev spaces by functions in finite element spaces has been well studied (cf. [3] and the references therein). One approach is to show the approximability of the nodal, finite element interpolant of a function. However, the nodal value of the function may not be well defined if the function under consideration is too "rough". For example, functions in the Sobolev space $H^{1}$ have no pointwise value in two or more dimensions. In [4], Clément defined an optimal-order interpolation operator using local averaging (regularizing) to define nodal values for functions even in $L^{1}$. However, the interpolator does not preserve homogeneous boundary conditions naturally. By setting boundary nodal values to zero, the modified interpolation operator posed in [4] can solve this problem, but this approach cannot be easily generalized to nonhomogeneous boundary data.

In this note, we propose another local averaging interpolation operator. We restrict our attention to simplicial finite elements, although this restriction is not essential. However, we consider general, $d$-dimensional meshes, $d \geq 2$; we assume only that the meshes are nondegenerate, i.e., we do not assume that they are quasi-uniform. Averaging is done either on a $d$-simplex or on a face $((d-1)$-simplex) of some $d$-simplex. This interpolation operator preserves homogeneous boundary conditions naturally. The key point is that this operator averages the interpolated functions on a subset (a $(d-1)$-simplex) of the boundary of the domain for each boundary nodal value, and it is of optimal order in approximation. We note that the interpolator is not defined for as broad a

Received March 24, 1989.

1980 Mathematics Subject Classification (1985 Revision). Primary 65D05; Secondary 65N30. 
class of functions as that of [4], but it is defined on functions smooth enough to have well-defined boundary values. A special case of this operator, interpolating $\stackrel{\circ}{H}^{1}$ functions by piecewise linear functions, was considered in [8]. We can also define an analogous interpolator for $L^{1}$ functions, using the techniques introduced here, that has properties similar to those of [4]. However, such an interpolator cannot be used to satisfy boundary conditions (such conditions are not well defined for $L^{1}$ functions in any case).

\section{INTERPOLATION}

Let $\Omega \subset \mathscr{E}^{d}$ be a connected, open, bounded, $d$-dimensional domain with polyhedral boundary. We assume that $\partial \Omega$ is Lipschitz continuous for simplicity, although many of the results presented can be extended easily to domains with simple slits. Let $C^{\infty}(\Omega)$ be functions defined on $\Omega$ and having continuous derivatives of any order, and let $\stackrel{\circ}{C}^{\infty}(\Omega)$ be functions in $C^{\infty}(\Omega)$ with compact support in $\Omega$. We denote by $L_{p}(\Omega)$ the completion of the functions in $C^{\infty}(\Omega)$ such that $\|f\|_{L_{p}(\Omega)}:=\left(\int_{\Omega}|f(x)|^{p} d x\right)^{1 / p}$ is finite, by $W_{p}^{l}(\Omega)$ the completion of the functions in $C^{\infty}(\Omega)$ such that $\|f\|_{W_{p}^{\prime}(\Omega)}:=\left(\sum_{|\alpha| \leq l}\left\|D^{\alpha} f\right\|_{L_{p}(\Omega)}^{p}\right)^{1 / p}$ is finite, and by $\stackrel{\circ}{W}_{p}^{l}(\Omega)$ the completion of the functions in $\stackrel{\circ}{C}^{\infty}(\Omega)$ such that $\|f\|_{W_{p}^{l}(\Omega)}$ is finite. Similarly, we define seminorms, $|f|_{W_{p}^{\prime}(\Omega)}:=\left(\sum_{|\alpha|=l}|| D^{\alpha} f \|_{L_{p}(\Omega)}^{p}\right)^{1 / p}$. Here, $\alpha=\left(\alpha_{1}, \ldots, \alpha_{d}\right)$ is a multi-index (each $\alpha_{i}$ is a nonnegative integer) with $|\alpha|:=\sum_{i=1}^{d} \alpha_{i}$ and $D^{\alpha /}:=\left(\partial / \partial x_{1}\right)^{\alpha_{1}} \cdots\left(\partial / \partial x_{d}\right)^{\alpha_{d}}$. We denote $W_{2}^{l}(\Omega)$ and $\stackrel{\circ}{W}_{2}^{l}(\Omega)$ by $H^{l}(\Omega)$ and $\stackrel{\circ}{H}^{l}(\Omega)$, respectively, as usual. For the definition of $W_{p}^{l}(\Omega)$ for fractional-order $l$, see [1].

Let $\mathscr{T}_{h}$ be a simplicial subdivision of $\Omega$ with maximum mesh size

$$
h:=\max _{K \in I_{h}} \operatorname{diam}(K)
$$

that is nondegenerate:

$$
\max _{K \in \mathcal{J}_{h}} \frac{\operatorname{diam}(K)}{\rho_{K}} \leq \gamma_{0}
$$

with the constant $\gamma_{0}$ independent of $h$. Note that we do not assume that all simplices, $K$, are of comparable size (that is, the mesh need not be quasiuniform). Here, $K$ denotes a $d$-simplex, $\operatorname{diam}(K)$ denotes the diameter of $K$ and $\rho_{K}$ denotes the radius of the largest closed ball contained in $\bar{K}$. For simplicity, we make the assumption that the spatial variable has been normalized so that $\operatorname{diam}(\Omega)=1$. We consider a finite element space $V_{h}$ consisting of continuous piecewise polynomials

$$
V_{h}:=\left\{v \in C(\Omega)|v|_{K} \in P_{K}=\mathscr{P}_{r}^{d} \forall K \in \mathscr{T}_{h}\right\}
$$


and the subspace $\stackrel{\circ}{V}_{h}$ consisting of continuous piecewise polynomials with homogeneous Dirichlet boundary condition:

$$
\stackrel{\circ}{V}_{h}:=\left\{v \in V_{h}|v|_{\partial \Omega}=0\right\} .
$$

Here, $\mathscr{P}_{r}^{d}$ is the space consisting of polynomials of degree $r$ or less in $d$ variables. For simplicity, we made the assumption $\mathscr{P}_{r}^{d}=P_{K}$ although the theorems in this paper can be extended to cover cases where $\mathscr{P}_{r}^{d} \subset P_{K}$. By (2.1), we have a family of Lagrange finite elements,

$$
\left(K, P_{K}, \Sigma_{K}\right), \quad K \in \mathscr{T}_{h},
$$

which are all affine equivalent to a single reference finite element $\left(\hat{K}, \mathscr{P}_{r}^{d}, \hat{\Sigma}\right)$ (cf. [3]). Here, $\hat{\Sigma}$ denotes the usual nodal variables for Lagrange interpolation consisting of point evaluations at appropriate points ("nodes") in $\hat{K}$, and $\Sigma_{K}$ denotes evaluations at points in $K$ that are the affine image of those for $\hat{\Sigma}$. For simplicity, we assume that $\hat{K}$ is a regular (equilateral) simplex having all edges of length one. We note that the theorems given in this paper also cover cases when the finite element spaces are of the form $\left\{v \in V_{h}|v|_{\Gamma}=0\right\}$ for some $\Gamma \subset \partial \Omega$, provided that the triangulation matches $\Gamma$ appropriately.

To define an interpolation operator on $W_{p}^{l}(\Omega)$, we make use of the nodal points, $\Sigma_{K}$, of the standard nodal interpolation operator. Let $\mathscr{N}_{h}=\left\{a_{i}\right\}_{i=1}^{N}$ be the set of all interpolation nodes of $\mathscr{T}_{h}$ and $\left\{\phi_{i}\right\}_{i=1}^{N}$ be the corresponding nodal basis of $V_{h}$. We choose, for any node $a_{i}$, either a $d$-simplex or a $(d-1)$ simplex, $\sigma_{i}$, according to the type of the node, $a_{i}$, as follows. If $a_{i}$ is an interior point of some $d$-simplex, $K \in \mathscr{T}_{h}$, we let

$$
\sigma_{i}=K \text {. }
$$

If $a_{i}$ is an interior point of some face (which is a $(d-1)$-simplex), $K^{\prime}$, of a $d$-simplex, $K$, we let

$$
\sigma_{i}=K^{\prime} .
$$

For the rest of the $a_{i} \in \mathcal{N}_{h}$, which must be on some $(d-2)$-simplex, there is considerable freedom in picking $\sigma_{i}$. We may pick any $(d-1)$-simplex, $K^{\prime}$, such that $a_{i} \in \overline{K^{\prime}}$, subject only to the restriction

$$
K^{\prime} \subset \partial \Omega \text { if } a_{i} \in \partial \Omega,
$$

and we set $\sigma_{i}=K^{\prime}$. We can see that the choice of $\sigma_{i}$ in (2.6) is not unique. The restriction $K^{\prime} \subset \partial \Omega$ in (2.6) for $a_{i} \in \partial \Omega$ is made for the purpose of preserving homogeneous boundary conditions.

For each face $(d-1)$-simplex, $K^{\prime}$, of $K$, there is a natural restriction of $\left(K, P_{K}, \Sigma_{K}\right)$ that defines a finite element:

$$
\left(K^{\prime}, P_{K^{\prime}}, \Sigma_{K^{\prime}}\right)=\left.\left(K, P_{K}, \Sigma_{K}\right)\right|_{K^{\prime}} .
$$


Here, $\Sigma_{K^{\prime}}$ consists of point evaluations at the points from $\Sigma_{K}$ that lie on the face $K^{\prime}$, and $P_{K^{\prime}}=\mathscr{P}_{r}^{d-1}$. For example, the restriction to one dimension of a linear, triangular, finite element would be a linear, line segment, finite element. Since all $\left(K, P_{K}, \Sigma_{K}\right)$ are affine equivalent to a single reference element, all $\left(K^{\prime}, P_{K^{\prime}}, \Sigma_{K^{\prime}}\right)$ are affine equivalent to a single, $(d-1)$-dimensional reference element. Further, the nonzero functions that are restrictions of the nodal basis functions of $P_{K}$ on $K^{\prime}$ comprise the nodal basis for $P_{K^{\prime}}$. Moreover, (2.1) implies that the set of all face $(d-1)$-simplices,

$$
\left\{K^{\prime} \mid K^{\prime} \text { is a face }((d-1) \text {-simplex }) \text { of } K \text {, for some } K \in \mathscr{T}_{h}\right\} \text {, }
$$

is a nondegenerate family of $(d-1)$-simplices. We remark that the converse of the last statement is false. For example, a sequence of tetrahedra having nondegenerate faces (with each face approaching a unit right triangle in the limit) can degenerate into a plane; the limit is a unit square with two diagonals.

Let us denote by $n_{1}$ the dimension of $\mathscr{P}_{r}^{d}$ and by $n_{0}\left(\sigma_{i}\right)$ the dimension of $\mathscr{P}_{r}^{\operatorname{dim} \sigma_{i}}$, i.e., either the dimension of $\mathscr{P}_{r}^{d-1}$ or $n_{1}$. Let $a_{i, 1}=a_{i}$, and let $\left\{a_{i, j}\right\}_{j=1}^{n_{0}}$ be the set of nodal points in $\sigma_{i}$. Here, $\sigma_{i}$ is defined in (2.4)-(2.6) associated with the node $a_{i}$. For the nodal basis $\left\{\phi_{i, j}\right\}_{j=1}^{n_{0}}$ for $\sigma_{i}$, we have an $L^{2}\left(\sigma_{i}\right)$-dual basis $\left\{\psi_{i, j}\right\}$ :

$$
\int_{\sigma_{i}} \psi_{i, j}(x) \phi_{i, k}(x) d x=\delta_{j k}, \quad j, k=1,2, \ldots, n_{0},
$$

where $\delta_{j k}$ is the Kronecker delta. For simplicity, we let

$$
\psi_{i}=\psi_{i, 1} \quad \forall a_{i} \in \mathscr{N}_{i} .
$$

Therefore, we have

$$
\int_{\sigma_{i}} \psi_{i}(x) \phi_{j}(x) d x=\delta_{i j}, \quad i, j=1,2, \ldots, N,
$$

where $\phi_{j}$ is any nodal basis function of $V_{h}$.

We define an interpolation operator,

$$
\Pi: W_{p}^{l}(\Omega) \rightarrow V_{h}(\Omega),
$$

by

$$
\Pi v(x)=\sum_{i=1}^{N} \phi_{i}(x) \int_{\sigma_{i}} \psi_{i}(\xi) v(\xi) d \xi
$$

where

$$
l \geq 1 \text { if } p=1 \text { and } l>1 / p \text { otherwise. }
$$

Here, $\Pi$ depends on the choices of $\sigma_{i}$ in (2.4)-(2.6), but we use the notation $\Pi$ instead of $\Pi_{\left\{\sigma_{i}\right\}}$ for simplicity. 
The condition (2.14) guarantees that the nodal values, $\left\{\Pi v\left(a_{i}\right)\right\}$, are well defined owing to the trace theorem (cf. [6] or [1]):

$$
\begin{aligned}
W_{p}^{l}(\Omega) \subset L^{1}\left(\sigma_{i}\right), \text { i.e., }\|v\|_{L^{\prime}\left(\sigma_{i}\right)} \leq C\left(\Omega, \sigma_{i}\right)\|v\|_{W_{p}^{\prime}(\Omega)}, & \\
& \forall v \in W_{p}^{l}(\Omega), i=1, \ldots, N .
\end{aligned}
$$

Further, the condition (2.14) guarantees the validity of the homogeneous boundary condition:

$$
\forall v \in \stackrel{\circ}{W}_{p}^{l}(\Omega),\left.\quad v\right|_{\partial \Omega}=0 \quad \text { in } L^{1}(\partial \Omega), \quad \text { i.e., }\|v\|_{L^{1}(\partial \Omega)}=0 .
$$

By the choice of $\sigma_{i}$ in (2.5), (2.6) we have, from (2.13) and (2.16), that

$$
v \in \stackrel{\circ}{W}_{p}^{l}(\Omega) \Longrightarrow \Pi v\left(a_{i}\right)=0 \quad \forall a_{i} \in \partial \Omega,
$$

and therefore that $\Pi$ preserves the homogeneous boundary condition:

$$
\Pi: \stackrel{\circ}{W}_{p}^{l}(\Omega) \rightarrow \stackrel{\circ}{V}_{h} .
$$

Since (2.11) implies that

$$
\int_{\sigma_{i}} \phi_{i}(x) v(x) d x=v\left(a_{i}\right) \quad \forall v \in V_{h},
$$

we conclude that $\Pi$ is a projection,

$$
\Pi v=v \quad \forall v \in V_{h} .
$$

We summarize the above results in the following theorem.

Theorem 2.1. Let $l$ and $p$ satisfy (2.14), and let $\mathscr{T}_{h}$ satisfy (2.1). Then the operator $\Pi$, defined in (2.13), is a projection from $W_{p}^{l}(\Omega)$ to $V_{h}$, defined in (2.2), with the property that $\stackrel{\circ}{W}_{p}^{l}(\Omega)$ is mapped to $\stackrel{\circ}{V}_{h}$, defined in (2.3).

\section{Stability}

In the rest of this paper, we will use " $\lesssim \ldots "$ and " $\simeq \ldots$ " to denote " $\leq C \ldots$ " and " $=C \ldots$ ", respectively, with the constant $C$ independent of the mesh $\mathscr{T}_{h}$ and independent of the functions under consideration.

Let $\left\{\sigma_{i}\right\}$ be a set of simplices that are either $d$-or $(d-1)$-simplices satisfying (2.1). Let $\hat{\sigma}$ be either the $d$-or the $(d-1)$-dimensional reference simplex, let $\left\{\hat{\phi}_{j}\right\}$ be a nodal basis for $\hat{\sigma}$ and let $\left\{\hat{\psi}_{j}\right\}$ be the dual basis with respect to the inner product for $L^{2}(\hat{\sigma})$. Suppose the affine mapping

$$
F(\hat{x})=B \hat{x}+x_{0}
$$


maps $\hat{\sigma}$ one-to-one and onto some $\sigma \in\left\{\sigma_{i}\right\}$; we then have (cf. [3])

$$
\begin{aligned}
\|B\| & \leq \frac{h_{\sigma}}{\hat{\rho}} \lesssim h_{\sigma}, \\
\left\|B^{-1}\right\| & \leq \frac{h_{\sigma}}{\rho_{\sigma}} \lesssim h_{\sigma}^{-1}, \\
\operatorname{det}(B) & =\frac{\operatorname{meas}(\hat{\sigma})}{\operatorname{meas}(\sigma)} \lesssim \begin{cases}h_{\sigma}^{d} & \text { if } \operatorname{dim}(\sigma)=d, \\
h_{\sigma}^{d-1} & \text { if } \operatorname{dim}(\sigma)=d-1 .\end{cases}
\end{aligned}
$$

Here $h_{\sigma}=\operatorname{diam}(\sigma)$ and $\rho_{\sigma}=\operatorname{diam}$ (the largest closed ball contained in $\bar{\sigma}$ ), with $\hat{h}$ and $\hat{\rho}$ defined correspondingly. Let $\left\{\bar{\phi}_{j}\right\}$ be the nodal basis, and $\left\{\bar{\psi}_{j}\right\}$ the dual basis to $\left\{\bar{\phi}_{j}\right\}$ on $\sigma$ :

$$
\int_{\sigma} \bar{\psi}_{j}(x) \bar{\phi}_{k}(x) d x=\delta_{j k} \quad \forall j, k .
$$

By the affine mapping defined in (3.1), it follows that

$$
\int_{\hat{\sigma}} \bar{\psi}_{j}\left(B \hat{x}+\hat{x}_{0}\right) \bar{\phi}_{k}\left(B \hat{x}+\hat{x}_{0}\right) \operatorname{det}(B) d \hat{x}=\delta_{j k} \quad \forall j, k .
$$

By the uniqueness of the dual basis and by noting $\hat{\phi}_{j}(\hat{x})=\bar{\phi}_{j}(F(\hat{x}))$, we see that

$$
\hat{\psi}_{j}=\operatorname{det}(B) \bar{\psi}_{j} \quad \forall j .
$$

By the regularity of the family $\left\{\sigma_{i}\right\}$ (both $d$ - and $(d-1)$-simplices), we get the following lemma by combining (3.3) and (3.2).

Lemma 3.1. For any node $a_{i} \in \mathcal{N}_{h}$,

$$
\left\|\psi_{i}\right\|_{L^{\infty}\left(\sigma_{i}\right)} \lesssim h_{K}^{-\operatorname{dim}\left(\sigma_{i}\right)}
$$

where $\sigma_{i}$ is the $d$-or $(d-1)$-simplex associated with $a_{i}$ defined in (2.4)-(2.6), $K \in \mathscr{T}_{h}$ is such that $\sigma_{i} \subset \bar{K}$, and $\psi_{i}$ is defined in (2.10).

Let $\sigma_{i}=K^{\prime}$ be a $(d-1)$-simplex that is the face of a $d$-simplex, $K$, and let $F$ defined in (3.1) map the reference $\hat{K}$ onto $K$ such that $F\left(\hat{K}^{\prime}\right)=K^{\prime}$ and $\hat{K}^{\prime}$ is the $(d-1)$-dimensional reference simplex. Here we assume that $\hat{K}^{\prime}$ is a face simplex of $\hat{K}$. Let us suppose that coordinates are chosen so that we can write

$$
\left.F\right|_{\hat{K}^{\prime}}\left(\hat{x}_{d-1}, 0\right)=\left(B_{d-1} \hat{x}_{d-1}, 0\right)+x_{0}
$$

(in particular, $\hat{K}^{\prime}$ lies in the plane $x_{d}=0$ ). It follows from the trace theorem 
and (3.2) that

$$
\begin{aligned}
\|v\|_{L^{\prime}\left(K^{\prime}\right)} & \leq\left|\operatorname{det}\left(B_{d-1}\right)\right|\|\hat{v}\|_{L^{\prime}\left(\hat{K}^{\prime}\right)} \lesssim\left|\operatorname{det}\left(B_{d-1}\right)\right|\|\hat{v}\|_{W_{p}^{\prime}(\hat{K})} \\
& \lesssim h_{K}^{d-1}\|\hat{v}\|_{W_{p}^{\prime}(\hat{K})} \lesssim h_{K}^{d-1} \sum_{k=0}^{l}\|B\|^{k}|\operatorname{det}(B)|^{-1 / p}|v|_{W_{p}^{k}(K)} \\
& \lesssim \sum_{k=0}^{l} h_{K}^{d-1+k-d / p}|v|_{W_{p}^{k}(K)} .
\end{aligned}
$$

Correspondingly, we have by Hölder's inequality that

$$
\|v\|_{L^{1}(K)} \lesssim h_{K}^{d-d / p}|v|_{W_{p}^{0}(K)} .
$$

Theorem 3.1. Let $v \in W_{p}^{l}(\Omega)$, let $l$ and $p$ satisfy $(2.14)$, let $\mathscr{T}_{h}$ satisfy (2.1) and let $K \in \mathscr{T}_{h}$. Then

$$
\|\Pi v\|_{W_{q}^{\prime m}(K)} \lesssim \sum_{k=0}^{l} h_{K}^{k-m+\frac{d}{q}-\frac{d}{p}}|v|_{W_{p}^{k}\left(S_{K}\right)},
$$

where

$$
S_{K}=\text { interior }\left(\bigcup\left\{\overline{K_{i}} \mid \overline{K_{i}} \cap \bar{K} \neq \varnothing, \quad K_{i} \in \mathscr{T}_{h}\right\}\right)
$$

and $\Pi$ is defined in (2.13). In (3.8), $1 \leq q \leq \infty$ and $m$ is any nonnegative integer.

Proof. Let $F$, defined in (3.1), map the reference simplex $\hat{K}$ onto $K$. We have, for any $v \in W_{q}^{m}(K)$,

$$
|v|_{W_{q}^{m}(K)} \lesssim\left\|B^{-1}\right\|^{m}|\operatorname{det}(B)|^{-\frac{1}{q}}|\hat{v}|_{W_{q}^{m}(\hat{K})} \lesssim h_{K}^{-m+\frac{d}{q}}|\hat{v}|_{W_{q}^{m}(\hat{K})}
$$

where $\hat{v}=v(F(\hat{x}))$. Further,

$$
\|v\|_{W_{q}^{m}(K)} \lesssim h_{K}^{-m+\frac{d}{q}}\|\hat{v}\|_{W_{q}^{m}(\hat{K})},
$$

since $h \leq \operatorname{diam}(\Omega)$. Without loss of generality, we may assume that the nodal points, $\left\{a_{i} \mid 1 \leq i \leq n_{1}\right\}$, comprise the nodal points for $\Sigma_{K}$. It follows then from (3.6)-(3.7) that, for any $v \in W_{p}^{l}(\Omega)$,

$$
\begin{aligned}
\|\Pi v\|_{W_{q}^{m}(K)} \leq \sum_{i=1}^{n_{1}}\left|\Pi v\left(a_{i}\right)\right|\left\|\phi_{i}\right\|_{W_{q}^{m}(K)} \lesssim h_{K}^{-m+\frac{d}{q}} \max _{1 \leq i \leq n_{1}}\left\|\hat{\phi}_{i}\right\|_{W_{q}^{m}(\hat{K})} \sum_{i=1}^{n_{1}}\left|\Pi v\left(a_{i}\right)\right| \\
\quad \lesssim h_{K}^{-m+\frac{d}{q}} \sum_{i=1}^{n_{1}}\left|\int_{\sigma_{i}} \psi_{i}(x) v(x) d x\right| \lesssim h_{K}^{-m+\frac{d}{q}} \sum_{i=1}^{n_{1}}\left\|\psi_{i}\right\|_{L^{\infty}\left(\sigma_{i}\right)}\|v\|_{L^{1}\left(\sigma_{i}\right)} \\
\quad \lesssim h_{K}^{-m+\frac{d}{q}} \sum_{i=1}^{n_{1}} h_{K}^{-\operatorname{dim}\left(\sigma_{i}\right)}\|v\|_{L^{1}\left(\sigma_{i}\right)} \lesssim h_{K}^{-m+\frac{d}{q}} \sum_{i=1}^{n_{1}} \sum_{k=0}^{l} h_{K}^{k-d / p}|v|_{W_{p}^{k}\left(K_{i}\right)} \\
\quad \sum_{k=0}^{l} h_{K}^{-m+\frac{d}{q}+k-\frac{d}{p}}|v|_{W_{p}^{k}\left(S_{K^{\prime}}\right)},
\end{aligned}
$$


where $K_{i}$ is a neighboring $d$-simplex such that $\sigma_{i} \subset \overline{K_{i}}$ and $S_{K}$ is defined in (3.9). Here, Lemma 3.1 was used to bound $\left\|\psi_{i}\right\|_{L^{\infty}\left(\sigma_{i}\right)}$, and we used the fact that (2.1) implies that $K, \sigma_{i}$, and $K_{i}$ are all of comparable size, i.e., that a nondegenerate mesh is locally quasi-uniform in two or more dimensions.

\section{APPROXIMABILITY}

We are going to consider $\|v-\Pi v\|_{S}$ for $v \in W_{p}^{l}(\Omega)$ and for various Sobolev norms, $\|\cdot\|_{S}$. For any $K \in \mathscr{T}_{h}$ and any polynomial $p \in \mathscr{P}_{r}^{d}$, we have by (2.18) and Theorem 3.1 that

$$
\begin{aligned}
\|v-\Pi v\|_{W_{p}^{m}(K)} & \leq\|v-p\|_{W_{p}^{m^{\prime}(K)}}+\|\Pi(p-v)\|_{W_{p}^{m}(K)} \\
& \leq \sum_{k=0}^{m} h_{K}^{k-m}\|v-p\|_{W_{p}^{k}\left(S_{K}\right)} .
\end{aligned}
$$

By (2.1), $S_{K}$ is the finite union of domains each of which is star-shaped with respect to a ball of radius $\rho$ times the diameter of $S_{K}$, with $\rho$ depending only on $\gamma_{0}$. Thus, we can apply the Bramble-Hilbert lemma in the form developed in [5, p. 458] to the right-hand side of (4.1) to obtain, for all $\forall v \in W_{p}^{l}\left(S_{K}\right)$,

$$
\inf _{p \in \mathscr{P}_{r}^{d}}\|v-p\|_{W_{p}^{k}\left(S_{K}\right)} \leq C\left(d, r, \gamma_{0}\right) h_{K}^{l-k}|v|_{W_{p}^{l}\left(S_{K}\right)}, \quad 0 \leq k \leq l \leq r+1 .
$$

In particular, we let the domains, $D_{j}$, in Theorem 7.1 of [5] be the interior of the closure of the union of pairs of simplices that share a common face. As a consequence of (2.1), such domains are each star-shaped with respect to a ball of radius $\rho$ times the diameter of $S_{K}$, where $\rho$ depends only on $\gamma_{0}$. Because of our assumption about the regularity of $\partial \Omega, S_{K}$ is connected. In view of Remark 7.2 of [5], (4.2) holds with a constant depending only on $d, r, \gamma_{0}$ because the intersection of a pair of domains, $D_{j}$, contains one of the simplices that make up $S_{K}$ and hence it contains a ball of radius $\tilde{\gamma}_{0}$ times the diameter of $S_{K}$, where $\tilde{\gamma}_{0}$ depends only on $\gamma_{0}$ in (2.1).

Combining (4.1) and (4.2) yields

$$
\|v-\Pi v\|_{W_{p}^{m}(K)} \leq C\left(d, r, \gamma_{0}\right) h_{K}^{l-m}|v|_{W_{p}^{\prime}\left(S_{K}\right)} .
$$

Since

$$
\sup _{\tilde{K} \in \mathscr{T}_{h}}\left\{\operatorname{card}\left\{K \in \mathscr{T}_{h} \mid \tilde{K} \cap S_{K} \neq \varnothing\right\}\right\}
$$

is a constant depending only on $\gamma_{0}$ in $(2.1)$, we easily deduce our main theorem.

Theorem 4.1. Let $v \in W_{p}^{l}(\Omega)$, let $l$ and $p$ satisfy (2.14), and let $\mathscr{T}_{h}$ satisfy (2.1). Then

$$
\left(\sum_{K \in \mathscr{T}_{h}} h_{K}^{p(m-l)}\|v-\Pi v\|_{W_{p}^{m}(K)}^{p}\right)^{1 / p} \leqslant\|v\|_{W_{p}^{\prime}(\Omega)}, \quad 0 \leq m \leq l \leq r+1,
$$

where $\Pi$ is defined in (2.13). 
Letting $m=l$ and applying the triangle inequality, the following corollary is derived.

Corollary 4.1. Let $v \in W_{p}^{l}(\Omega)$, let $l$ and $p$ satisfy (2.14), and let $\mathscr{T}_{h}$ satisfy (2.1). Then

$$
\left(\sum_{K \in T_{h}}\|\Pi v\|_{W_{p}^{\prime}(K)}^{p}\right)^{1 / p} \lesssim\|v\|_{W_{p}^{\prime}(\Omega)},
$$

where $\Pi$ is defined in (2.13).

Recalling that $h=\max _{K \in \mathcal{F}_{h}} \operatorname{diam}(K)$, the statment of Theorem 4.1 can be simplified as follows:

$$
\left(\sum_{K \in \mathcal{F}_{h}}\|v-\Pi v\|_{W_{p}^{\prime \prime}(K)}^{p}\right)^{1 / p} \lesssim h^{l-m}\|v\|_{W_{p}^{\prime}(\Omega)}, \quad 0 \leq m \leq l \leq r+1 .
$$

Note that the results obtained so far easily extend to domains with simple slits. In fact, the critical condition is that the set $S_{K}$ in (3.9) be connected, and this can be arranged in such a case. We also note that if one is not interested in preserving boundary conditions, the techniques used here can also be used to define interpolators with optimal-order approximation properties for functions only in $L^{1}(\Omega)$. In such a case, the $\sigma_{i}$ 's are all chosen to be $d$-dimensional simplices containing the corresponding points, $a_{i}$. That is, the nodal values for all nodal points, $a_{i}$, are defined in the way that the nodal values for nodal points in the interior of each simplex were defined, cf. (2.4). In this way, one obtains an interpolator with properties similar to those of [4].

\section{APPLiCATIONS}

One way that the projection operator (2.13) can be used is to treat the Dirichlet problem for an elliptic boundary value problem,

$$
\begin{gathered}
-\sum_{i, j=1}^{d} \frac{\partial}{\partial x_{j}}\left(\alpha_{i j} \frac{\partial u}{\partial x_{i}}\right)=0 \text { in } \Omega, \\
u=g \text { on } \partial \Omega .
\end{gathered}
$$

Here we assume the $\alpha_{i j}$ are bounded, measurable functions on $\Omega$ which form a uniformly positive definite matrix a.e. on $\Omega$. Assuming $g$ to be defined on all of $\Omega$, and smooth enough for an interpolant, $\Pi g$, to be defined, then it is natural to define an approximant to $u$ by seeking $u_{h}$ in the space

$$
V_{h}^{g}:=\left\{v \in V_{h} \mid v=\Pi g \text { on } \partial \Omega\right\}=\left\{v \in V_{h} \mid v-\Pi g \in \stackrel{\circ}{V}_{h}\right\}
$$


such that

$$
a\left(u_{h}, v\right):=\int_{\Omega} \sum_{i, j=1}^{d} \alpha_{i j} \frac{\partial u_{h}}{\partial x_{i}} \frac{\partial v}{\partial x_{j}} d x=0 \quad \forall v \in \stackrel{\circ}{V}_{h} .
$$

Note that $u_{h}$ depends only on $\left.\Pi g\right|_{\partial \Omega}$. If $\Pi$ is the interpolator defined in (2.13), $\left.\Pi g\right|_{\partial \Omega}$ depends only on $\left.g\right|_{\partial \Omega}$. In particular, $u$ furnishes an extension to $\left.g\right|_{\partial \Omega}$, so we may think of $u_{h}$ being defined using the space

$$
V_{h}^{g}=\left\{v \in V_{h} \mid v-\Pi u \in \stackrel{\circ}{V}_{h}\right\},
$$

since $\left.\Pi g\right|_{\partial \Omega}=\left.\Pi u\right|_{\partial \Omega}$. Note that not all interpolators, e.g. [4], would necessarily have this property.

We can derive error estimates using standard techniques in the natural energy norm

$$
\|v\|_{E}^{2}:=a(v, v)
$$

Since $a\left(u_{h}, v\right)=a(u, v)=0$ for $v \in \stackrel{\circ}{V}_{h}$, we have

$$
\left\|\Pi u-u_{h}\right\|_{E}^{2}=a\left(\Pi u-u_{h}, \Pi u-u_{h}\right)=a\left(\Pi u, \Pi u-u_{h}\right)=a\left(\Pi u-u, \Pi u-u_{h}\right) .
$$

Applying Schwarz's inequality, we find

$$
\left\|\Pi u-u_{h}\right\|_{E} \leq\|\Pi u-u\|_{E} .
$$

From the triangle inequality we thus have

$$
\left\|u-u_{h}\right\|_{E} \leq 2\|u-\Pi u\|_{E} .
$$

The error estimate (4.6) therefore implies that

$$
\begin{array}{r}
\left\|u-u_{h}\right\|_{E} \lesssim \max \left\{\left\|\alpha_{i j}\right\|_{L^{\infty}(\Omega)} \mid i, j=1, \ldots, d\right\} h^{l-1}\|u\|_{H^{\prime}(\Omega)}, \\
1 \leq l \leq r+1 .
\end{array}
$$

For estimates in other Sobolev norms, see [7]. We note that $\Pi g$ can be defined when standard (pointwise) interpolants may not be defined, e.g., for unbounded or discontinuous $g$. Moreover, $\Pi g$ can be evaluated locally, so that the implementation of such a method is straightforward. Our techniques can be viewed as providing a systematic way of "averaging" the boundary data in such cases.

Another application of the interpolator defined in (2.13) is to extension of boundary data. Let us assume that $d=2$ for simplicity. One can define Sobolev spaces $W_{p}^{s}(\partial \Omega)$ for real values of $s \in[0,1]$ provided that $\partial \Omega$ is Lipschitz continuous, as we are assuming. Furthermore, there is a continuous extension operator, $E: W_{p}^{1-1 / p}(\partial \Omega) \longrightarrow W_{p}^{1}(\Omega)$ for $1 \leq p<\infty$ (cf. [2] and references therein). Composing with $\Pi$, we obtain a map, $E_{h}=\Pi E: W_{p}^{1-1 / p}(\partial \Omega) \longrightarrow$ $V_{h}$, having the property that

$$
\left\|E_{h} v\right\|_{W_{p}^{\prime}(\Omega)} \lesssim\|v\|_{W_{p}^{1-1 / p}(\partial \Omega)} .
$$


Moreover, let $\Gamma$ be any subset of $\partial \Omega$, and define

$$
\Gamma_{h}=\bigcup\left\{\sigma_{i} \mid \sigma_{i} \subset \Gamma\right\},
$$

where $\sigma_{i}$ is defined in (2.4)-(2.6). If $v$ is a piecewise polynomial on $\Gamma$ of degree $r$, then (2.11) implies that $E_{h} v=v$ on $\Gamma_{h}$. Thus we have an extension operator that maps piecewise polynomials on (parts of) the boundary to piecewise polynomials on $\Omega$, that is bounded as a map from $W_{p}^{1-1 / p}(\partial \Omega)$ to $W_{p}^{1}(\Omega)$.

\section{BIBLIOGRAPHY}

1. R. A. Adams, Sobolev spaces, Academic Press, New York, 1975.

2. D. N. Arnold, L. R. Scott, and M. Vogelius, Regular inversion of the divergence operator with Dirichlet boundary conditions on a polygon, Ann. Scuola Norm. Sup. Pisa Cl. Sci. (4) 15 (1988), 169-192.

3. P. G. Ciarlet, The finite element method for elliptic problems, North-Holland, Amsterdam, 1978.

4. P. Clement, Approximation by finite element functions using local regularization, RAIRO Anal. Numér. R-2 (1975), 77-84.

5. T. Dupont and R. Scott, Polynomial approximation of functions in Sobolev spaces, Math. Comp. 34 (1980), 441-463.

6. J. Nečas, Les méthodes directes en théorie des équations elliptiques, Academia, Prague, 1967.

7. P. Saavedra and L. R. Scott, $A$ variational formulation of free boundary problems, submitted to Math. Comp.

8. S. Zhang, Multi-level iterative techniques, thesis, Pennsylvania State University, 1988.

Department of Mathematics, Pennsylvania State University, University Park, PennSYLVANIA 16802

Department of Mathematics, Purdue University, West Lafayette, Indiana 47907 